03

\title{
Математическое моделирование конверсии углеводородного топлива в элементах теплозащиты гиперзвуковых летательных аппаратов
}

\author{
() А.Л. Куранов, А.В. Корабельников, А.М. Михайлов
}

Научно-исследовательское предприятие гиперзвуковых систем, 196066 Санкт-Петербург, Россия

e-mail: mail@hypersonics.ru

(Поступило в Редакцию 14 апреля 2016 г.)

Представлена математическая модель процесса конверсии углеводородного топлива внутри термохимического реактора как элемента тепловой защиты гиперзвукового летательного аппарата. Применение модели позволило дополнить информацию, полученную в экспериментальных исследованиях.

DOI: 10.21883/JTF.2017.01.44014.1856

\section{Введение}

В последние годы многие промышленно развитые страны активизировали работы по созданию гиперзвуковых летательных аппаратов (ГЛА) на углеводородном топливе [1]. Использование углеводородного топлива в таких аппаратах возможно только после его преобразования (конверсии) перед подачей в камеру сгорания [2]. При конверсии углеводородного топлива образуется водородосодержащий газ, улучшающий экологические и энергетические характеристики горения. В элементах теплозащиты камеры сгорания и особо теплонапряженных участков обшивки планера используют реакции конверсии, обладающие значительным эндотермическим эффектом (до $200 \mathrm{~kJ} / \mathrm{mol}$ ).

Элементы теплозащиты представляют собой термохимические реакторы (ТХР), в которых протекают различные химические процессы. В настоящей работе рассмотрена каталитическая реакция паровой конверсии метана $\left(\mathrm{CH}_{4}+\mathrm{H}_{2} \mathrm{O} \rightarrow 3 \mathrm{H}_{2}+\mathrm{CO}\right)$ как основного компонента разложения жидкого углеводородного топлива керосина [3]. Использование метана в качестве компонента реакции паровой конверсии позволяет оценить предельные возможности ТХР по теплопоглощению и количеству получаемого водорода [4].

В настоящей работе представлены аспекты математического моделирования ТХР с целью восстановления ряда экспериментальных параметров, которые не могли быть измерены приборным способом. На основании этих данных получены удельные характеристики процессов, достигнутые в ходе экспериментов.

\section{Экспериментальное исследование}

Проведение экспериментов имело целью определение режимов работы ТХР теплозащиты с максимальной степенью конверсии при условии снятия значительных тепловых потоков. Кроме того, проверялась применимость выбранного материала корпуса ТХР и анализировалась работа новых катализаторов.
На стенде для тепловых испытаний ТХР был реализован масс-спектрометрический анализ состава газа в нескольких точках по длине реактора.

Геометрически центральный канал реактора был выполнен в форме прямоугольника с шириной, значительно превосходящей высоту. В такой конструкции отпадает необходимость защиты боковых стенок, удаленных от центра потока. Два канала ТХР, в которые подается смесь метана и водяного пара, были размещены около самых теплонапряженных поверхностей. На рис. 1 изображена принципиальная схема экспериментального TХР. На входе в ТХР был размещен смеситель с тангенциальной подачей компонентов, обеспечивающей смешение и распределение их между верхним и нижним каналами.

Для увеличения степени конверсии углеводородного топлива были созданы специальные каркасные катализаторы. Полученный катализатор представляет собой спрессованную нихромовую проволоку, вытравленную для повышения удельной поверхности и активированную в кислородной атмосфере в муфельной печи. Более подробное описание экспериментальной установки представлено в [5].

Входными параметрами эксперимента являлись: расходы водяного пара и метана в реакторе, расход азота в плазмотроне, температура смеси паров воды и метана

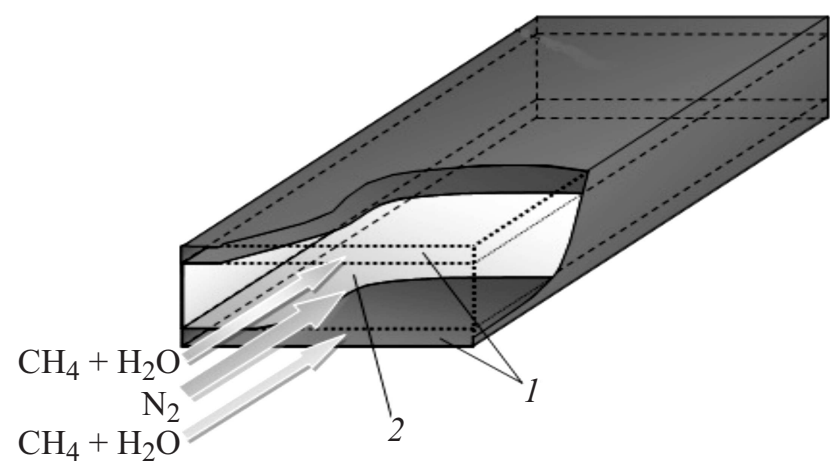

Рис. 1. Схема экспериментального ТХР: 1 - центральный канал; 2 - каналы ТХР. 
Таблица 1. Основные параметры экспериментов

\begin{tabular}{c|c|c|c|c}
\hline Режим & $\begin{array}{c}\text { Расход } \\
\text { водяного } \\
\text { пара, g/s }\end{array}$ & $\begin{array}{c}\text { Расход } \\
\text { метана, g/s }\end{array}$ & $\begin{array}{c}\text { Мощность } \\
\text { плазмотрона, } \\
\mathrm{kW}\end{array}$ & $\begin{array}{c}\text { Средняя } \\
\text { температура } \\
\text { стенки, K }\end{array}$ \\
\hline 1.1 & 0.27 & 0.13 & 25.1 & 1277 \\
2.1 & 0.3 & 0.13 & 14.8 & 838 \\
2.2 & 0.29 & 0.14 & 39.5 & 1255 \\
2.3 & 1 & 0.51 & 49.8 & 1514 \\
3.1 & 0.26 & 0.12 & 16.3 & 1011 \\
3.2 & 0.26 & 0.13 & 29.9 & 1330 \\
4.1 & 0.26 & 0.13 & 26.1 & 1247 \\
4.2 & 0.44 & 0.33 & 30.1 & 1250 \\
4.3 & 0.44 & 0.54 & 30 & 1271 \\
4.4 & 0.45 & 0.53 & 51 & 1453 \\
4.5 & 0.45 & 0.54 & 60.2 & 1502
\end{tabular}

на входе в реактор, мощность плазмотрона. Основными непосредственно измеряемыми выходными параметрами были температура охлаждаемой стенки реактора, определяемая в шести точках, и химический состав смеси, отбираемый в точках пробоотбора.

На основании измеренных величин входных и выходных параметров были получены величина теплового потока, скорость течения смеси и степень конверсии метана. Всего было проведено четыре продолжительных эксперимента, в ходе которых исследовано 11 стационарных режимов. Во всех экспериментах расход азота поддерживался на уровне $9.1 \mathrm{~g} / \mathrm{s}$, а температура смеси водяного пара и метана на входе в реактор была близкой к $600 \mathrm{~K}$. Остальные параметры экспериментов указаны в табл. 1. В первом эксперименте в каналы ТХР не был установлен каркасный катализатор. Более подробно проведение экспериментальных исследований рассмотрено в [6].

\section{Математическое моделирование}

Для полного описания процессов тепломассообмена недостаточно экспериментальных данных, полученных при проведении эксперимента, так как не все физические величины поддаются непосредственному или косвенному измерению. Для определения недостающих данных необходимо использовать аппарат математического моделирования. Основываясь на полученных в ходе эксперимента данных, можно составить модель, описывающую соотношение искомых величин в условиях протекавшего эксперимента.

В первую очередь были определены величины удельного теплового потока, исходящего от азотной плазмы и поглощаемого в ТХР в течение эксперимента. Поглощаемый удельный тепловой поток является основным критерием применимости паровой конверсии в элементах тепловой защиты. Для экспериментов, в которых не было получено данных о составе газа, была рассчитана степень конверсии и определена относительная эффек- тивность работы ТХР с каталитическим наполнением в реализованных режимах.

Для определения ненаблюдаемых параметров была разработана дополняющая модель, описывающая центральный продольный вертикальный срез модельного TXP, поскольку все датчики температуры располагались именно в плоскости этого среза.

Первая часть модели (модель центрального канала) описывает взаимодействие струи азотной плазмы и металлической стенки ТХР с известной измеренной температурой, в этой части определяются величины удельного теплового потока. Вторая часть модели (модель ТХР) описывает процессы, происходящие непосредственно внутри ТХР, а именно нагрев и химические преобразования потока компонентов. В этой части модели определяются скорость и состав реагирующей смеси, расчет производится на основании вычисленного удельного теплового потока.

Математические расчеты велись в среде конечноразностного моделирования - Comsol 4.3a. В первой части модели эксперимента использовался модуль Conjugate Heat Transfer, описывающий кондуктивный и конвективный теплообмены в движущихся по ламинарным законам средах и твердых телах. Во второй части модели использовались модули Darcy's Low, Heat Transfer in Fluids и Transport of Concentrated Species. Совокупность данных модулей описывает теплообмен и термохимические процессы для многокомпонентной смеси, протекающей в пористой среде.

Исследуемые режимы являются стационарными по задающим параметрам, но в целом система не находится в термодинамическом равновесии. Исходя из этого, расчеты необходимо проводить во временной области. Начальным моментом времени был выбран момент включения расхода метана при включенном плазмотроне и расходе водяного пара. Для определения начальных условий были рассчитаны стационарные случаи, обусловленные значениями параметров в момент до пуска метана.

\section{Модель центрального канала}

Геометрически модель центрального канала представляет собой канал $\mathrm{ABCD}$ длиной $571 \mathrm{~mm}$ и шириной $20 \mathrm{~mm}$ со стенками $\mathrm{AA}^{\prime} \mathrm{D}^{\prime} \mathrm{D}$ и $\mathrm{BB}^{\prime} \mathrm{C}^{\prime} \mathrm{C}$ толщиной $4 \mathrm{~mm}$ (рис. 2). Через край $\mathrm{AB}$ в канал поступает разогретый в плазмотроне азот. Протекая по центральному каналу, азот нагревает стенки.

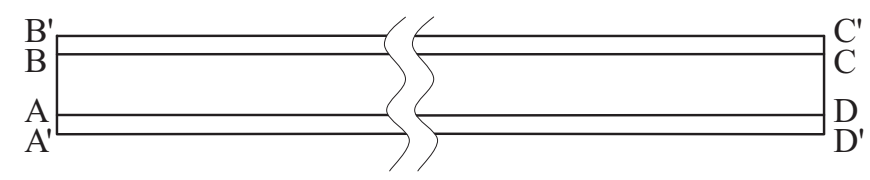

Рис. 2. Геометрическое представление модели центрального канала. 
Поток азота задается массовым расходом через плазмотрон, для всех экспериментов этот параметр фиксировался на уровне $9.1 \mathrm{~g} / \mathrm{s}$. Нагрев азота происходит тепловым потоком, равным электрической мощности плазмотрона за вычетом потерь на охлаждение. Число $\operatorname{Re}$ в центральном канале определяется как

$$
\operatorname{Re}=\frac{G_{\mathrm{N}_{2}} \cdot d_{r}}{F \cdot \mu_{\mathrm{N}_{2}}},
$$

где $G_{\mathrm{N}_{2}}-$ массовый расход азота, $\mathrm{kg} / \mathrm{s} ; d_{r}-$ гидравлический диаметр канала, $\mathrm{m} ; F-$ площадь сечения канала, $\mathrm{m}^{2} ; \mu_{\mathrm{N}_{2}}-$ вязкость азота, $\mathrm{kg} /(\mathrm{m} \cdot \mathrm{s})$.

$\operatorname{Re}$ во всех режимах $<700$, что говорит о возможности применения ламинарной модели течения. Параметры азота были взяты из [7]. Параметры материала стенок центрального канала были взяты из [8]. Температура охлаждаемых стенок была определена интерполированием показаний пристеночных термопар в каждый момент времени.

Течение и теплообмен в центральном канале ABCD описывается системой уравнений

$$
\left\{\begin{array}{l}
\rho \frac{\partial \mathbf{u}}{\partial t}+\rho(\mathbf{u} \times \nabla) \mathbf{u}=\nabla \times\left(-\rho I+\mu\left(\nabla \mathbf{u}+(\nabla \mathbf{u})^{T}\right)\right)+\mathbf{F}, \\
\rho \boldsymbol{\nabla} \times \mathbf{u}=0, \\
\rho C_{p} \frac{\partial T}{\partial t}+\rho C_{p} \mathbf{u} \times \nabla T=\nabla \times(k \nabla T)+Q,
\end{array}\right.
$$

где $\rho$ - плотность, $\mathrm{kg} / \mathrm{m}^{3} ; \mathbf{u}-$ вектор скорости, $\mathrm{m} / \mathrm{s}$; $t$ - время, s; $I$ - единичная матрица; $\mu$ - динамическая вязкость, Рa·s; $\mathbf{F}$ - градиент давления, $\mathrm{Pa} / \mathrm{m}$; $C_{p}$ - теплоемкость при постоянном давлении, $\mathrm{J} /(\mathrm{K} \cdot \mathrm{kg})$; $k$ - теплопроводность, $\mathrm{W} /(\mathrm{m} \cdot \mathrm{K}) ; Q-$ тепловой поток, W. B этой системе первое уравнение - уравнение Навье-Стокса в векторной форме, второе - уравнение изменения плотности, третье - уравнения кондуктивного и конвективного теплообменов.

Граничными условиями в $\mathrm{BC}$ и $\mathrm{AD}$ является

$$
\mathbf{u}=0 \text {. }
$$

Граничные условия в АВ определяют поток азота $m, \mathrm{~g} / \mathrm{s}$ :

$$
-d_{b c} \int_{\partial \Omega} \rho(\mathbf{u} \times \mathbf{n}) d S=m
$$

и спутный с ним тепловой поток $q_{0}, \mathrm{~W}$ :

$$
\begin{aligned}
-\mathbf{n} \times(-k \nabla T)= & \left(-q_{0}(\mathbf{u} \times \mathbf{n}) \frac{\int l}{\int|\mathbf{u} \times \mathbf{n}|}\right) \\
& +\rho\left(\Delta h_{i n}-\Delta h_{\infty}\right) \mathbf{u} \times \mathbf{n},
\end{aligned}
$$

где $\Delta h_{i n}-\Delta h_{\infty}=\int_{T_{\infty}}^{T_{i n}} C_{p} d T+\int_{P_{\infty}}^{P T_{a}} \frac{1}{\rho}\left(1+\left.\frac{T}{\rho}\left(\frac{\partial \rho}{\partial T}\right)\right|_{p}\right) d p$.

Граничные условия в $\mathrm{CD}$ определяют свободное истечение азота в среду с давлением $p_{0}=100 \mathrm{kPa}$ :

$$
p=p_{0}
$$

$$
\left(\mu\left(\nabla \mathbf{u}+(\nabla \mathbf{u})^{T}\right)-\frac{2}{3} \mu(\nabla \times \mathbf{u}) \mathbf{l}\right) \times \mathbf{n}=0 .
$$

Кроме этого, в CD определяется выход тепла только в качестве теплоты азота

$$
-\mathbf{n} \times(-k \nabla T)=0 .
$$

Теплообмен в стенках $\mathrm{AA}^{\prime} \mathrm{D}^{\prime} \mathrm{D}$ и $\mathrm{BB}^{\prime} \mathrm{C}^{\prime} \mathrm{C}$ описывается уравнением теплообмена

$$
\rho C_{p} \frac{\partial T}{\partial t}+\rho C_{p} \mathbf{u} \times \nabla T=\nabla \times(k \nabla T)+Q .
$$

Краевыми условиями для стенок является: теплоизолированность стенок $\mathrm{AA}^{\prime}, \mathrm{BB}^{\prime}, \mathrm{CC}^{\prime}$ и $\mathrm{DD}^{\prime}$

$$
-\mathbf{n} \times(-k \nabla T)=0,
$$

а для $\mathrm{A}^{\prime} \mathrm{D}^{\prime}$ и $\mathrm{B}^{\prime} \mathrm{C}^{\prime}$ - температура, соответствующая температуре стенки при испытании натурной модели, определенная по интерполяции экспериментальных данных:

$$
T=T_{0}(t, x)
$$

\section{Модель канала термохимического реактора}

В двумерном приближении ТХР представляет собой прямоугольный канал (рис. 3), по которому от сечения EF к сечению GH протекает реагирующая смесь. Канал заполнен пористым катализатором. Состав смеси меняется по мере срабатывания исходных компонентов. Если на входе смесь состоит из метана и водяного пара, то на выходе основными компонентами являются водород и монооксид углерода. Стенка FG теплоизолирована, а через стенку ЕН, температура которой соответствует температуре стенки натурной модели при эксперименте, протекает тепловой поток, определяемый первой частью математической модели эксперимента.

При расчете расход компонентов - водяного пара и метана - равен расходам в течение эксперимента, температура на входе в реактор равна измеренной. Свойства среды и компонентов приведены в табл. 1. Термодинамические свойства газов взяты из [7], свойства водяного пара из [9], кинетические характеристики протекания химической реакции и коэффициенты диффузии взяты из [10], коэффициент проницаемости определен на основании экспериментальных данных по формуле

$$
k_{\mathrm{per}}=\frac{Q \cdot \mu \cdot L}{\Delta P \cdot F}
$$

где $Q$ - объемный расход флюида, $\mathrm{mm}^{3} / \mathrm{s} ; \mu-$ вязкость флюида, sPu; $\Delta P$ - перепад давления, $\mathrm{Pa} ; F-$ площадь

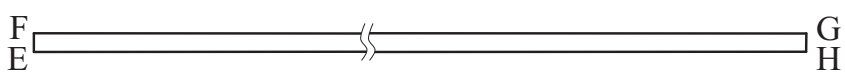

Рис. 3. Геометрическое представление модели ТХР. 
Таблица 2. Поглощаемый тепловой поток

\begin{tabular}{|c|c|c|c|c|c|c|}
\hline \multirow{3}{*}{$\begin{array}{c}\text { Режим, время с начала } \\
\text { эксперимента, с }\end{array}$} & \multicolumn{6}{|c|}{ Величина теплового потока, kW/m² } \\
\hline & $\begin{array}{c}\text { точка } \\
1\end{array}$ & $\begin{array}{c}\text { точка } \\
2\end{array}$ & $\begin{array}{c}\text { точка } \\
3\end{array}$ & $\begin{array}{c}\text { точка } \\
4\end{array}$ & $\begin{array}{c}\text { точка } \\
5\end{array}$ & $\begin{array}{c}\text { точка } \\
6\end{array}$ \\
\hline & \multicolumn{6}{|c|}{$\begin{array}{c}\text { Модель центрального канала } \\
\text { (расчет в приближении круглого канала и равномерного } \\
\text { распределения температуры) }\end{array}$} \\
\hline & \multicolumn{6}{|c|}{ Первый эксперимент } \\
\hline \multirow[t]{2}{*}{$1.1,2600$} & 9.2 & 4.5 & 1.2 & 1.1 & 2 & 3.7 \\
\hline & \multicolumn{6}{|c|}{ Второй эксперимент } \\
\hline $2.1,1000$ & $\begin{array}{l}9.4 \\
5.1\end{array}$ & $\begin{array}{l}6.1 \\
3.4\end{array}$ & $\begin{array}{l}4.6 \\
4.2\end{array}$ & $\begin{array}{l}4.3 \\
3.8\end{array}$ & $\begin{array}{l}4.1 \\
3.3\end{array}$ & $\begin{array}{l}4.2 \\
3.1\end{array}$ \\
\hline $2.2,1700$ & $\begin{array}{r}20.3 \\
19\end{array}$ & $\begin{array}{c}12.1 \\
12\end{array}$ & $\begin{array}{r}7.5 \\
11.3\end{array}$ & $\begin{array}{l}6.7 \\
7.6\end{array}$ & $\begin{array}{l}6.7 \\
7.2\end{array}$ & $\begin{array}{l}7.8 \\
6.1\end{array}$ \\
\hline \multirow[t]{2}{*}{$2.3,2150$} & $\begin{array}{l}51.5 \\
49.8\end{array}$ & $\begin{array}{l}35.7 \\
30.4\end{array}$ & $\begin{array}{l}27.7 \\
22.5\end{array}$ & $\begin{array}{l}23.3 \\
16.4\end{array}$ & $\begin{array}{l}20.7 \\
11.2\end{array}$ & $\begin{array}{r}21.4 \\
9.6\end{array}$ \\
\hline & \multicolumn{6}{|c|}{ Третий эксперимент } \\
\hline $3.1,4825$ & $\begin{array}{l}8.2 \\
6.1\end{array}$ & $\begin{array}{l}5.2 \\
2.9\end{array}$ & $\begin{array}{l}3.7 \\
3.6\end{array}$ & $\begin{array}{l}3.6 \\
2.5\end{array}$ & $\begin{array}{l}3.8 \\
1.9\end{array}$ & $\begin{array}{l}4.3 \\
1.8\end{array}$ \\
\hline \multirow[t]{2}{*}{$3.2,5900$} & $\begin{array}{l}20.3 \\
19.4\end{array}$ & $\begin{array}{l}13.3 \\
11.5\end{array}$ & $\begin{array}{r}10.1 \\
9.6\end{array}$ & $\begin{array}{l}9.5 \\
7.9\end{array}$ & $\begin{array}{l}9.1 \\
5.1\end{array}$ & $\begin{array}{l}9.5 \\
4.9\end{array}$ \\
\hline & \multicolumn{6}{|c|}{ Четвертый эксперимент } \\
\hline $4.1,2700$ & $\begin{array}{l}18.2 \\
13.1\end{array}$ & $\begin{array}{c}9.4 \\
6\end{array}$ & $\begin{array}{l}6.9 \\
5.9\end{array}$ & $\begin{array}{l}6.4 \\
5.7\end{array}$ & $\begin{array}{l}6.1 \\
4.7\end{array}$ & $\begin{array}{l}6.5 \\
3.4\end{array}$ \\
\hline $4.2,3800$ & $\begin{array}{c}30 \\
20.8\end{array}$ & $\begin{array}{c}17 \\
11.1\end{array}$ & $\begin{array}{l}11 \\
8.7\end{array}$ & $\begin{array}{r}10.8 \\
7.8\end{array}$ & $\begin{array}{r}10.9 \\
4.8\end{array}$ & $\begin{array}{r}11.5 \\
4.3\end{array}$ \\
\hline $4.3,4300$ & $\begin{array}{l}31.4 \\
21.6\end{array}$ & $\begin{array}{l}17.2 \\
11.7\end{array}$ & $\begin{array}{r}11.4 \\
8.8\end{array}$ & $\begin{array}{l}11 \\
7.9\end{array}$ & $\begin{array}{r}10.9 \\
4.1\end{array}$ & $\begin{array}{c}11.2 \\
4\end{array}$ \\
\hline $4.4,4750$ & $\begin{array}{l}53.6 \\
50.7\end{array}$ & $\begin{array}{c}38.7 \\
32\end{array}$ & $\begin{array}{l}30.2 \\
24.1\end{array}$ & $\begin{array}{l}27.7 \\
18.3\end{array}$ & $\begin{array}{l}27.1 \\
12.7\end{array}$ & $\begin{array}{r}28.4 \\
9.9\end{array}$ \\
\hline $4.5,4825$ & $\begin{array}{l}69.9 \\
64.1\end{array}$ & $\begin{array}{l}40.6 \\
41.8\end{array}$ & $\begin{array}{l}29.3 \\
31.2\end{array}$ & $\begin{array}{l}27.3 \\
23.7\end{array}$ & $\begin{array}{c}26.5 \\
16\end{array}$ & $\begin{array}{l}25.5 \\
13.2\end{array}$ \\
\hline
\end{tabular}

фильтрации, $\mathrm{mm}^{2} ; L$ - длина образца, $\mathrm{mm}$. Коэффициент пористости определен по разности истинной и удельной плотности.

Протекающие в реакторе процессы описываются системой уравнений

$$
\left\{\begin{array}{l}
\frac{\partial}{\partial t}\left(\rho \varepsilon_{p}\right)+\nabla(\rho \mathbf{u})=Q_{m}, \\
\rho C_{p} \frac{\partial T}{\partial t}+\rho C_{p} \mathbf{u} \times \nabla T=\nabla \times(k \nabla T)+Q, \\
\rho \frac{\partial \omega_{i}}{\partial t}+\nabla \times \mathbf{j}_{i}+\rho(\mathbf{u} \times \nabla) \omega_{i}=R_{i},
\end{array}\right.
$$

где: $\quad \mathbf{u}=-\frac{k_{\text {per }}}{\mu} \frac{\nabla \rho}{\nabla} ; \quad \mathbf{j}_{i}=-\left(\rho D_{i}^{m} \nabla \omega_{i}+\rho \omega_{i} D_{i}^{m} \frac{\nabla_{M_{n}}}{M_{n}}+\right.$ $\left.+D_{i}^{T} \frac{\nabla_{T}}{t}\right) ; \mathbf{N}_{i}=\mathbf{j}_{i}+\rho \mathbf{u} \omega_{i} ; D_{i}^{m}=\frac{1-\omega_{i}}{\sum_{k=i} \frac{x_{k}}{D_{i k}}} ; M_{n}=\left(\sum_{i} \frac{\omega_{i}}{M_{i}}\right)^{-1} ;$ $t$ - время, $\mathrm{s} ; \rho-$ плотность, $\mathrm{kg} / \mathrm{m}^{3} ; \varepsilon_{p}$ - коэффициент пористости; и - вектор скорости, m/s; $Q_{m}$ - тепловой поток от реакции, $\mathrm{W} ; C_{p}-$ теплоемкость при постоянном давлении, $\mathrm{J} /(\mathrm{K} \cdot \mathrm{kg}) ; \quad T-$ температура, $\mathrm{K} ; \quad k_{\text {per }}-$ коэффициент проницаемости, $\mathrm{mm}^{2}$; $k$ - теплопроводность, $\mathrm{W} /(\mathrm{m} \cdot \mathrm{K}) ; \omega_{i}-$ массовая доля компонента $i ; \mathbf{j}_{i}-$ мощность источника $i$-го компонента, кg/s; $R_{i}$ - массовая скорость реакции, $\mathrm{Kg} /\left(\mathrm{m}^{3} \cdot \mathrm{s}\right) ; D_{i k}$ - коэффициент взаимной диффузии $i$-го и $k$-го компонентов, $\mathrm{m}^{2} / \mathrm{s} ; D_{i}^{T}-$ коэффициент термодиффузии, $\mathrm{kg} /(\mathrm{m} \cdot \mathrm{s}) ; x_{k}-$ объемная доля $k$-го компонента; $M_{i}$ - молярная масса $i$-го компонента, $\mathrm{g} / \mathrm{mol}$.

Первое уравнение в системе - уравнение закона Дарси, второе - уравнение теплообмена, третье закон динамики каждого компонента смеси. 
Таблица 3. Относительные концентрации компонентов газа

\begin{tabular}{|c|c|c|c|c|c|c|c|}
\hline \multirow{2}{*}{$\begin{array}{c}\text { Место } \\
\text { отбора проб } \\
\text { (мм от начала } \\
\text { реактора) }\end{array}$} & \multirow{2}{*}{$\begin{array}{l}\text { Время } \\
\text { отбора }\end{array}$} & \multicolumn{3}{|c|}{$\begin{array}{c}\text { Результаты масс-спектро- } \\
\text { метрического анализа }\end{array}$} & \multicolumn{3}{|c|}{ Расчетные концентрации } \\
\hline & & $\mathrm{H}_{2}$ & $\mathrm{CH}_{4}$ & $\mathrm{CO}$ & $\mathrm{H}_{2}$ & $\mathrm{CH}_{4}$ & $\mathrm{CO}$ \\
\hline 153 & 2097 & 0.43 & 0.42 & 0.14 & 0.43 & 0.43 & 0.14 \\
\hline 332 & 2151 & 0.56 & 0.25 & 0.19 & 0.55 & 0.26 & 0.19 \\
\hline 421 & 2274 & 0.63 & 0.16 & 0.21 & 0.64 & 0.15 & 0.21 \\
\hline 501 & 2346 & 0.71 & 0.06 & 0.23 & 0.71 & 0.06 & 0.23 \\
\hline 501 & 2427 & 0.7 & 0.07 & 0.23 & 0.71 & 0.06 & 0.24 \\
\hline 559 & 2496 & 0.74 & 0.02 & 0.24 & 0.74 & 0.02 & 0.24 \\
\hline 559 & 2547 & 0.73 & 0.03 & 0.24 & 0.73 & 0.03 & 0.24 \\
\hline 153 & 3063 & 0.21 & 0.72 & 0.07 & 0.19 & 0.75 & 0.06 \\
\hline 332 & 3135 & 0.47 & 0.37 & 0.16 & 0.47 & 0.37 & 0.16 \\
\hline 332 & 3363 & 0.47 & 0.38 & 0.15 & 0.46 & 0.4 & 0.14 \\
\hline 501 & 3480 & 0.65 & 0.14 & 0.21 & 0.66 & 0.13 & 0.21 \\
\hline 421 & 3630 & 0.53 & 0.29 & 0.18 & 0.56 & 0.25 & 0.19 \\
\hline 153 & 4095 & 0.04 & 0.96 & 0.01 & 0.05 & 0.95 & 0.01 \\
\hline 332 & 4227 & 0.34 & 0.55 & 0.11 & 0.33 & 0.56 & 0.11 \\
\hline 421 & 4296 & 0.44 & 0.41 & 0.15 & 0.44 & 0.41 & 0.15 \\
\hline 501 & 4386 & 0.67 & 0.11 & 0.22 & 0.66 & 0.12 & 0.22 \\
\hline 559 & 4512 & 0.74 & 0.01 & 0.25 & 0.75 & 0 & 0.25 \\
\hline 153 & 4626 & 0.1 & 0.87 & 0.03 & 0.12 & 0.85 & 0.04 \\
\hline 332 & 4674 & 0.19 & 0.75 & 0.06 & 0.2 & 0.74 & 0.06 \\
\hline 421 & 4716 & 0.58 & 0.23 & 0.19 & 0.6 & 0.2 & 0.19 \\
\hline 501 & 4743 & 0.74 & 0.02 & 0.24 & 0.75 & 0.01 & 0.24 \\
\hline 559 & 4809 & 0.75 & 0 & 0.25 & 0.75 & 0.01 & 0.25 \\
\hline 153 & 4857 & 0.21 & 0.72 & 0.07 & 0.22 & 0.7 & 0.07 \\
\hline 332 & 4881 & 0.42 & 0.44 & 0.14 & 0.42 & 0.44 & 0.14 \\
\hline 421 & 4890 & 0.48 & 0.36 & 0.16 & 0.48 & 0.36 & 0.16 \\
\hline 501 & 4941 & 0.68 & 0.09 & 0.23 & 0.68 & 0.09 & 0.23 \\
\hline 559 & 4983 & 0.74 & 0.01 & 0.25 & 0.73 & 0.02 & 0.25 \\
\hline 559 & 5000 & 0.75 & 0 & 0.25 & 0.74 & 0.01 & 0.25 \\
\hline
\end{tabular}

Граничные условия на ЕН описываются системой

$$
\left\{\begin{array}{l}
-\mathbf{n} \times \rho \mathbf{u}=0, \\
-\mathbf{n} \times(-k \nabla T)=q_{0}, \\
-\mathbf{n} \times \mathbf{N}_{i}=0
\end{array}\right.
$$

где $q_{0}$ - тепловой поток от центрального канала.

Граничные условия на EF описываются системой

$$
\left\{\begin{array}{l}
-\mathbf{n} \times \rho \mathbf{n}=N_{0}, \\
T=T_{i n}, \\
\omega_{i}=\omega_{0 i},
\end{array}\right.
$$

где $T_{i n}$ - температура входящей смеси, $N_{0}-$ расход азота, $\omega_{0 i}$ - начальная массовая доля компонентов.

Граничные условия на FG описываются системой

$$
\left\{\begin{array}{l}
-\mathbf{n} \times \rho \mathbf{u}=0, \\
-\mathbf{n} \times(-k \nabla T)=0, \\
-\mathbf{n} \times \mathbf{N}_{i}=0 .
\end{array}\right.
$$

Граничные условия на GH описываются системой

$$
\left\{\begin{array}{l}
p=p_{0}, \\
-\mathbf{n} \times(-k \nabla t)=0, \\
-\mathbf{n} \times \rho D_{i}^{m} \nabla \omega=0,
\end{array}\right.
$$

где $p_{0}$ - давление на выходе.

Для расчета использовалась сетка с непостоянным размером ячеек, определяемым интенсивностью изменения физических параметров. В качестве начального условия для расчета использовалось состояние натурной модели до момента запуска плазмотрона. Расчет каждого шага производился методом MUMPS (MUltifrontal Massively Parallel sparse direct Solver) с нелинейными ньютоновскими шагами. Шаги по времени осуществлялись методом формул обратного дифференцирования.

Для расчета использовались данные экспериментов I, II, III и IV, а именно показания датчиков температуры стенки, датчиков тока и напряжения плазмотрона, температуры и расхода воды охлаждения, расхода азота, метана, показания комплекса измерения расхода водяного пара, температуры смеси на входе в ТХР. Для всех 
Таблица 4. Удельное теплопоглощение и степень использования хладоресурса

\begin{tabular}{|c|c|c|c|c|c|c|}
\hline Режим & $\begin{array}{c}\text { Удельное } \\
\text { теплопоглощение } \\
\text { эксперимен- } \\
\text { тальное, } \mathrm{Mj} / \mathrm{kg}\end{array}$ & $\begin{array}{c}\text { Удельный } \\
\text { хладоресурс, Mj/kg }\end{array}$ & $\begin{array}{c}\text { Степень } \\
\text { использования } \\
\text { хладоресурса } \\
\text { внутри ТХР, \% }\end{array}$ & $\begin{array}{c}\text { Удельный выход } \\
\text { водорода, g/kg } \\
\text { исходной смеси }\end{array}$ & $\begin{array}{c}\text { Выход } \\
\text { водорода } \\
\text { молярный }\end{array}$ & $\begin{array}{c}\text { Степень } \\
\text { конверсии }\end{array}$ \\
\hline 1.1 & 2.42 & 5.53 & 44 & 52 & 0.35 & 0.43 \\
\hline 2.1 & 0.42 & 5.14 & 8 & 10 & 0.08 & 0.11 \\
\hline 2.2 & 3.02 & 5.54 & 55 & 119 & 0.61 & 0.98 \\
\hline 2.3 & 1.82 & 5.74 & 32 & 125 & 0.62 & 0.98 \\
\hline 3.1 & 0.57 & 5.37 & 11 & 13 & 0.10 & 0.14 \\
\hline 3.2 & 3.56 & 5.67 & 63 & 123 & 0.62 & 0.98 \\
\hline 4.1 & 2.04 & 5.96 & 34 & 130 & 0.64 & 0.98 \\
\hline 4.2 & 1.74 & 7.39 & 24 & 140 & 0.66 & 0.86 \\
\hline 4.3 & 1.45 & 8.81 & 17 & 168 & 0.72 & 0.99 \\
\hline 4.4 & 2.88 & 9.01 & 32 & 177 & 0.73 & 0.99 \\
\hline 4.5 & 3.25 & 9.19 & 35 & 180 & 0.73 & 0.99 \\
\hline
\end{tabular}

параметров методом сплайн-интерполяции показаний датчиков была определена временная функциональная зависимость. Также определено распределение температур по длине реактора на основании показаний датчиков температуры стенки.

\section{Результаты расчета}

В результате проведенных расчетов были получены изменения значений теплового потока в течение эксперимента по длине реактора. Сравнение значений теплового потока, полученных различными методами в стационарных режимах, представлено в табл. 2.

Как видно из табл. 2, разные методы расчета дали близкие результаты, но стоит отметить, что в области малых значений величина теплового потока, полученная при расчете, применявшемся при проведении эксперимента, значительно отличается от рассчитанной по модели центрального канала. Эта разница обусловлена различиями в математическом представлении центрального канала в методах расчета.

В применявшемся при эксперименте методе расчета, канал представляется одномерной моделью взаимодействия потока со стенкой. При этом к измеренной температуре стенки приравнивается температура всего сечения, что является не очень точным допущением при геометрии канала, далекой от осесимметричной. В конечно-разностном расчете центральный канал описывается моделью центрального сечения. При этом не учитывается отток тепла в поперечном направлении от центрального продольного сечения, где интенсивность теплообмена в силу геометрии канала наибольшая.

Поскольку модель центрального канала обусловлена экспериментальными данными и при этом результаты, полученные с помощью этой модели, имеют высокую степень соответствия результатам расчетов по другим

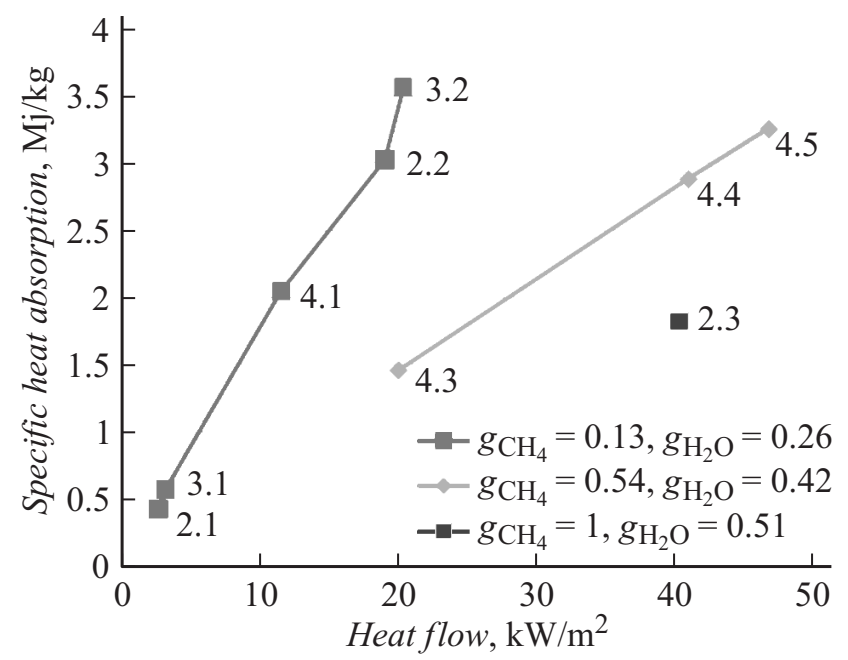

Рис. 4. Зависимость удельного теплопоглощения от величины теплового потока.

моделям, то именно ее можно принять, как наиболее достоверную для расчета величины теплового потока.

Для того чтобы охарактеризовать изменение расчетного состава реагирующего газа по длине и по времени, было рассчитано усреднение значений относительных концентраций компонентов. При сопоставлении расчетных и экспериментальных результатов наблюдалась схожесть результатов.

В табл. 3 представлены результаты масс-спектрометрического анализа и расчетных значений концентрации компонентов в соответствующих точках в одинаковых условиях.

Различия в составах обусловлены множеством факторов, среди которых неравномерное усреднение смеси при отборе проб, не учитывающиеся в модели тепловые потери, неточность задания модели протекания химической реакции и т.д. Важным полученным параметром 


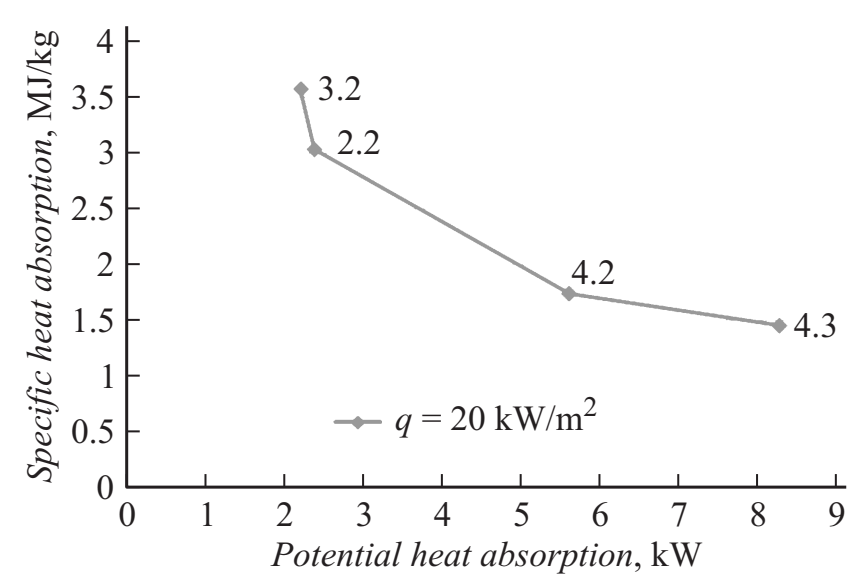

Рис. 5. Зависимость удельного теплопоглощения от расхода хладоресурса.

является удельное теплопоглощение, которое определяется как величина тепловой энергии, поглощенной единицей массы реагирующей смеси, и в стационарном режиме рассчитывается по следующей формуле:

$$
\omega=\frac{s \cdot \int_{0}^{l} q(x) d x}{G},
$$

где $q(x)$ - удельный тепловой поток, $\mathrm{W} / \mathrm{m}^{2}$, на расстоянии $x, \mathrm{~m}$, от начала реактора; $l$ - длина реактора, $\mathrm{m}$; $s$ - ширина реактора, $\mathrm{m} ; G-$ массовый расход компонентов, $\mathrm{kg} / \mathrm{s}$.

Для исследованных временных зависимостей параметров были определены величины, соответствующие стационарным, устоявшимся режимам. В плоскости задающих параметров - теплового потока и расходуемого хладоресурса были реализованы три запланированные серии экспериментов. В первой серии экспериментов величина расхода хладоресурса находилась в пределах $2.2 \pm 0.2 \mathrm{~kW}$, при этом тепловой поток изменялся в интервале $2-20 \mathrm{~kW} / \mathrm{m}^{2}$, во второй $-8.6 \pm 0.4 \mathrm{~kW}$ при изменении теплового потока в интервале $20-47 \mathrm{~kW} / \mathrm{m}^{2}$. B третьей серии величина теплового потока была $20 \pm 0.5 \mathrm{~kW} / \mathrm{m}^{2}$ при изменении расхода хладоресурса в интервале $2.2-8.3 \mathrm{~kW}$.

При постоянных параметрах смеси для одного состава определен линейный характер зависимости полученного удельного теплопоглощения от величины теплового потока (рис. 4), в то время как изменение состава при таком же общем расходе хладоресурса привело к снижению удельного теплопоглощения (сравнение 4.4 и 2.3 на рис. 4).

При постоянном тепловом потоке была определена зависимость удельного теплопоглощения от расхода хладоресурса (рис. 5).

Экспериментальные и теоретические величины удельного теплопоглощения показаны в табл. 4. Там же показана величина удельного выхода водорода.

\section{Заключение}

Из соотношения удельного теплопоглощения и удельного хладоресурса можно сделать вывод, что непосредственно от охлаждаемой стенки смесь поглощает всего от 17 до 63\% общего хладоресурса в режимах с высокой степенью конверсии (0.86-0.99). Это объясняется тем, что на этапе подготовки и смешения компоненты поглощают достаточно тепла для осуществления высокой степени конверсии, а тепло, получаемое от стенки, хоть и вносит существенный вклад в тепловой баланс, но не значительно влияет на степень конверсии. Наибольшая величина удельного выхода водорода - более $165 \mathrm{~g} / \mathrm{kg}$ исходной смеси в экспериментах - достигается с расходом метана большим, чем расход воды.

\section{Список литературы}

[1] Бальмина Р.В., Губанов А.А., Иванькин М.А., Лапинский Д.А. // Состояние и перспективы разработки гиперзвукового вооружения. Новости зарубежной науки и техники. Серия: авиационная и ракетная техники. 2012. Вып. 1-2. С. $1821-1822$.

[2] Lander H., Nixon A. // AIAA. 1968. T. 997. C. 1-12.

[3] Куранов А.Л., Корабельников А.В., Михайлов А.М. Принципы управления и моделирования тепловой защиты гиперзвукового летательного аппарата. СПб: Изд-во политех. ун-та, 2014. 227 с.

[4] Михайлов А.М. // Научно-технические ведомости СПбГПУ. 2013. № 3 (178). С. 95-100.

[5] Куранов А.Л., Корабельников А.В., Михайлов А.М. // Письма в ЖТФ. 2014. Т. 40. Вып. 2. С. 90-94.

[6] Куранов А.Л., Корабельников А.В., Михайлов А.М. // 5th Europ. Conf. Aeronautics and Space Sciences (eucass): Abstracts. Munich, 2013. ISBN: 978-84-941531-0-5

[7] Варгафтик Н.Б. Справочник по теплофизическим свойствам газов и жидкостей. М.: Наука, 1972. 720 с.

[8] Масленков С.Б. Жаропрочные стали и сплавы. Справочное издание. М.: Металлургия, 1983. 192 с.

[9] Александров А.А., Григорьев Б.А. // Таблицы теплофизических свойств воды и водяного пара. Справочник. М.: Изд-во МЭИ, 1999. 168 с.

[10] Новый справочник химика и технолога. Электродные процессы. Химическая кинетика и диффузия. Коллоидная химия / Под общ. ред. С.А. Симановой. СПб.: АНО НПО Профессионал, 2004. 838 с. 